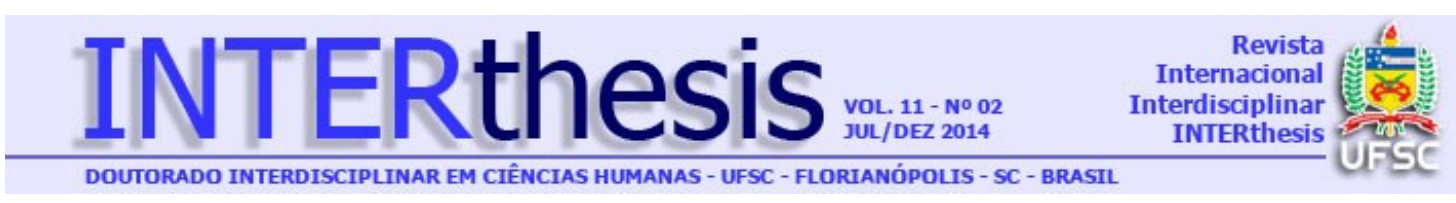

\title{
SOBRE EL ORIGEN DEL CONCEPTO DE “INMANENCIA" EN GILLES DELEUZE
}

\author{
Marcelo Sebastián Antonelli ${ }^{1}$
}

\section{Resumen}

El concepto de "inmanencia" desempeña un rol fundamental en la filosofía de Gilles Deleuze. Si bien suele destacarse su noción de "plano de inmanencia", el pensador francés elaboró un enfoque integral sobre este concepto, conectándolo con problemas políticos (el capitalismo es descripto como un sistema inmanente) y éticos (la moral universal es sustituida por una ética de valores inmanentes). Los comentaristas han reparado en las distintas formulaciones del concepto, no obstante lo cual no han investigado aún, de manera exhaustiva, el modo en que aparece por primera vez en su obra. En razón de ello, nos proponemos en este trabajo analizar el contexto de origen de la idea deleuziana de inmanencia, que involucra aspectos filosóficos y filológicos. De acuerdo con nuestra hipótesis, la primera aparición del concepto se sitúa en Spinoza et le problème de l'expression (1968), donde nuestro autor inscribe a Spinoza en la tradición de la univocatio entis iniciada por Duns Escoto. Sostenemos que la inmanencia deleuziana abreva en dos perspectivas: la tesis escotista de la univocatio entis frente a la analogia entis de filiación aristotélicotomista y la causa sui espinoziana frente a la causa emanativa neoplatónica y la causa analógica tomista. La fórmula "Inmanencia = Univocidad", puesta de relieve por Badiou, expresa este anudamiento conceptual que hemos de desarrollar. Desde el punto de vista filológico, mostraremos que Deleuze acuña el giro "ser inmanado", al momento de describir la especificidad de la causa inmanente, a partir de un uso peculiar de los verbos del latín manare y manere.

Palabras-clave: Deleuze. Duns Escoto. Spinoza. Inmanencia. Univocidad.

\section{INTRODUCCIÓN: SOBRE EL CONCEPTO DE INMANENCIA EN DELEUZE}

El concepto de "inmanencia" forma parte de la tradición filosófica occidental desde sus inicios. Su origen se remonta a Aristóteles (1998, pp. 54-55; 64-65); su uso se prolonga en el medioevo (MAGNAVACCA, 2005, p. 344) y llega hasta el siglo XX (LALANDE, 1968, pp. 468 ss.; ABBAGNANO, 1995, p. 678 ss.). El término ha

\footnotetext{
1 Doctor en Filosofía pela Universidade de Buenos Aires, Investigador Asociado al Centro de Investigaciones Filosóficas, Profesor Adjunto regular en la Universidad Pedagógica de la Provincia de Buenos Aires, Buenos Aires, Argentina. E-mail: antonelli.ms@gmail.com
}

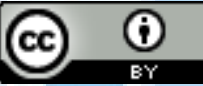
Esta obra foi licenciada com uma Licença Creative Commons - Atribuição 3.0 Não Adaptada. 
adquirido una creciente relevancia, al punto que ha sido empleado como un criterio ordenador de la filosofía moderna (AGAMBEN, 1998, p. 187; CICCARELLI, 2008).

En el caso de Deleuze, la inmanencia constituye uno de los conceptos fundamentales de su filosofía. Si bien suele destacarse su idea de "plano de inmanencia", nuestro autor elaboró un enfoque integral sobre la noción, conectándola con problemas políticos (el capitalismo es descripto como un sistema inmanente; DELEUZE y GUATTARI, 1973, pp. 271 ss., 274, 280, 282 ss., 298 ss., 311-312, 314 ss., 400, 414, 440 ss.; 2006, p. 579) y éticos (la moral basada en valores universales es sustituida por una ética anclada en valores inmanentes; DELEUZE, 1968, pp. 234-251; 1983, pp. 27-43). A nuestro juicio, la reivindicación irrestricta de la inmanencia, junto con la recusación de toda trascendencia, conforma el núcleo de la apuesta filosófica deleuziana.

La noción ha sido estudiada por diversos comentadores (BADIOU, 1997; DE BEISTEGUI, 2005; KERSLAKE, 2009; MENGUE, 1994; MONTEBELLO, 2008; SHIRANI, 2007; SMITH, 2012), no obstante lo cual su origen en la obra deleuziana no ha sido analizado aún de manera exhaustiva. La idea de inmanencia no aparece por primera vez como "plano de inmanencia" ni como "inmanencia capitalista", sino en la fórmula "ser inmanado" en el marco del panteísmo expresivo espinosista.

En este trabajo nos proponemos desarrollar el contexto, que involucra aspectos filosóficos y filológicos, en el cual Deleuze presenta dicha enunciación. Nuestra hipótesis es que la inmanencia deleuziana deriva de dos posiciones ontológicas diferentes, desde la perspectiva de la historia de la filosofía, pero reunidas por nuestro autor al elaborar el concepto: en primer lugar, la tesis escotista de la univocatio entis frente a la analogia entis de filiación aristotélico-tomista y, en segundo lugar, la causa sui espinoziana frente a la causa emanativa neoplatónica y la causa analógica tomista. El origen de la noción deleuziana se sitúa en la convergencia de estas tesis: ella sintetiza a la vez el carácter unívoco del ser y la producción causal cuyo efecto no trasciende su agente sino que permanece en él. La fórmula "Inmanencia = Univocidad", que Deleuze le habría escrito a Badiou (1998, p. 28) durante el intercambio epistolar que mantuvieron pocos años antes de su muerte, expresa este anudamiento conceptual que nos proponemos desplegar.

Desde un punto de vista historiográfico, el concepto aparece por primera vez, como ha notado Agamben (1998, p. 172), en Spinoza et le problème de l'expression.

R. Inter. Interdisc. INTERthesis, Florianópolis, v.11, n.2, p.15-36, Jul-Dez. 2014
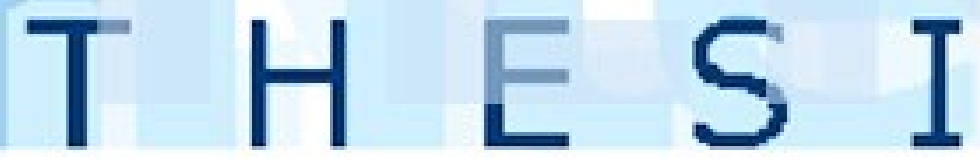
La conjunción de Duns Escoto y Spinoza, poco explorada antes del texto deleuziano, da como resultado una concepción de la inmanencia que adopta de Spinoza sus elementos fundamentales pero los pasa a través de la criba escotista. Según nuestro autor, la teoría inmanente de la expresión se despoja de los elementos ligados a la equivocidad y la analogía cuando se conjuga con la determinación del ser como unívoco. Dicho de otro modo, la inscripción de Spinoza en el linaje de la univocidad hace posible que el autor de la Ética arribe a la auténtica inmanencia en la idea de "causa inmanente", comprendida como proceso de inmanación.

Cabe aclarar que no abordaremos las múltiples dimensiones de la lectura deleuziana de Spinoza, sino que nos concentraremos en los aspectos de su interpretación ligados a nuestro objeto de estudio. Nuestro recorrido seguirá el siguiente orden: primero, nos detendremos en la inscripción de Spinoza en la tradición del expresionismo (§ II. 1); luego, precisaremos en qué sentidos Deleuze lo concibe como un pensador de la univocidad (§ II. 2.; $\S$ II. 3.); por último, profundizaremos la formación histórica de una inmanencia expresiva, lo cual nos permitirá analizar la especificidad de la elaboración deleuziana de la idea de inmanencia (§ II. 4.)

\section{CLAVES DE LA INTERPRETACIÓN DELEUZIANA DE SPINOZA}

\subsection{INMANENCIA Y EXPRESIONISMO}

Una primera consideración concierne el problema de determinar si, como sostiene Deleuze, Spinoza es efectivamente un filósofo de la inmanencia, esto es, si hay un "concepto espinosista de inmanencia" (DELEUZE, 1968, p. 150). Al respecto, cabe observar que, desde un punto de vista filológico, el término no es utilizado literalmente por el autor de la Ética, no obstante lo cual su empleo es consistente desde el punto de vista filosófico. Como marca Chaquet (2010),

cuando [Deleuze] define [a Spinoza] como filósofo de la inmanencia, le hace decir lo que no dice pero que sin embargo está presente en él. En efecto, la inmanencia, hablando con rigor, no es un concepto espinosista. Spinoza no emplea jamás este sustantivo, sino sólo el adjetivo "inmanente" para calificar la causalidad de la sustancia. Por tanto, la idea se encuentra

R. Inter. Interdisc. INTERthesis, Florianópolis, v.11, n.2, p.15-36, Jul-Dez. 2014
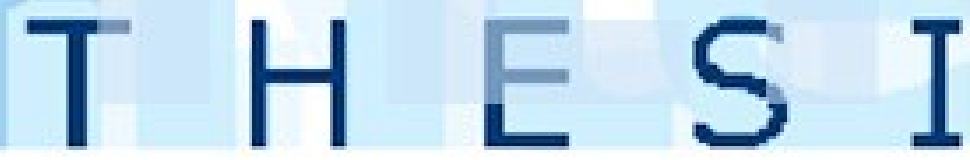
presente, sin estar formulada como tal y revestir la radicalidad que le es adjudicada. $^{2}$

El contexto en el cual surge la interpretación deleuziana de Spinoza en tanto pensador de la inmanencia es su inscripción en las tradiciones de la expresión y de la univocidad. Veremos a continuación, en primer lugar, los lineamientos fundamentales de la problemática de la expresión. Se trata de un enfoque preliminar que será retomado más adelante, cuando presentemos la explicación deleuziana del proceso histórico de formación de una "inmanencia expresiva" -cf. infra § II. 4.

Nuestro autor presenta su lectura de Spinoza como una historia del "expresionismo" en filosofía y despliega el sistema espinosista a la luz de la problemática de la "expresión". ${ }^{3}$ El objetivo de Deleuze es determinar cómo se inserta Spinoza en dicha tradición, qué elementos toma y cómo la renueva. El interés por la expresión obedece no sólo a la importancia que tendría en el primer libro de la Ética, sino a que remite a una tradición filosófica acusada de panteísmo:

\begin{abstract}
El concepto de expresión [...] es un concepto propiamente filosófico, de contenido inmanente, que se inmiscuye (immisce) en los conceptos trascendentes de una teología emanativa o creacionista. Lleva consigo el "peligro" propiamente filosófico: el panteísmo o la inmanencia -inmanencia de la expresión en lo que se expresa, y de lo expresado en la expresión- [...] nacido en las tradiciones de la emanación y de la creación, él se hace de dos enemigos, porque enfrenta tanto la trascendencia de un Uno superior al ser como la trascendencia de un Ser superior a la creación (DELEUZE, 1968, pp. 300).
\end{abstract}

La idea de expresión tiene como términos correlativos explicare e involvere, que mientan su doble aspecto: la expresión engloba o implica lo que expresa, al mismo tiempo que lo explica y desarrolla (cf. DELEUZE, 1968, p. 12). Deleuze sostiene que la implicación y la explicación no se oponen sino que remiten a un

\footnotetext{
2 Las traducciones son nuestras en todos los casos.

${ }^{3}$ Macherey (1996, p. 141) subraya la originalidad de la lectura de Deleuze en la medida en que el concepto de "expresión" resulta, a primera vista, ajeno al proyecto espinosista. En efecto, la forma nominal espressio no aparece nunca como tal en la Ética, no obstante lo cual la idea de "expresión" es sugerida a través del uso del verbo exprimere, empleado de diversas maneras (expressa, exprimatur, exprimere, exprimerem, exprimet, exprimit, exprimunt, exprimuntur). En consecuencia, pese a la ausencia del sustantivo, la tesis de Deleuze se vería apoyada por dicho uso de las formas verbales. Por otra parte, el autor observa que en Spinoza: philosophie pratique Deleuze no incluye el término "expresión" en el glosario de los principales conceptos de la Ética, lo cual lo lleva a pensar que ella cumplía "un rol derivado"; se trataba, más bien, de un "un dispositivo retórico" necesario para la construcción argumental, pero finalmente redundante (cf. MACHEREY, 1996, p. 145). De todas maneras, Macherey justifica el planteo deleuziano pues afirma que, aun si Spinoza no consideró la idea de expresión en cuanto tal, confrontó el problema mismo de la expresión, que se halla efectivamente presente en su obra. Por su parte, Hardt (1993, p. 68 y ss.) observa que la teoría de la expresión deleuziana es un desafío al juicio de Hegel según el cual el espinosismo es "una concepción oriental de la emanación" consistente en una serie de degradaciones.
}

R. Inter. Interdisc. INTERthesis, Florianópolis, v.11, n.2, p.15-36, Jul-Dez. 2014
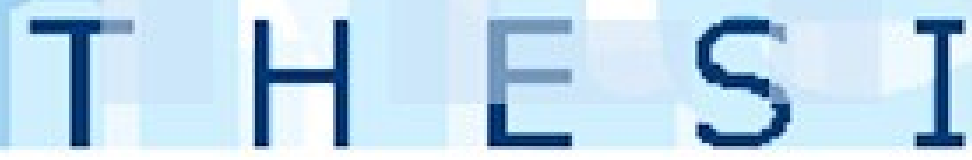
mismo principio sintético, la complicatio, que designaba en el neoplatonismo la presencia de lo múltiple en el Uno y del Uno en lo múltiple. En el contexto espinosista, la expresión resume las dificultades concernientes a la unidad de la substancia y la diversidad de los atributos, lo Uno y lo Múltiple (DELEUZE, 1968, p. 9). La fórmula que pretende resolver este punto es "ontológicamente uno, formalmente diverso", lo cual significa que "la multiplicidad de los atributos es estrictamente igual a la unidad de la substancia [...] los atributos son formalmente lo que la substancia es ontológicamente" (DELEUZE, 1968, p. 309).

En este marco, Deleuze entiende por "inmanencia" el panteísmo expresivo espinosista, al cual alude de modo indistinto como "el panteísmo o la inmanencia", "el panteísmo o la inmanencia expresiva” (DELEUZE, 1968, pp. 300, 310). El concepto espinosista de inmanencia remite para nuestro autor, en primer lugar, al de Naturaleza: la Naturaleza naturante, como substancia y causa, y la Naturaleza naturada, como modo y efecto, mantienen un lazo de mutua inmanencia, en tanto la causa permanece en sí para producir (a diferencia de la causa transitiva) y el efecto permanece en la causa (a diferencia de la causa emanativa). Profundizaremos, más adelante, este carácter singular de la causa inmanente -cf. infra § II. 4.

\subsection{SPINOZA, PENSADOR DE LA UNIVOCIDAD}

Como adelantamos, Deleuze inscribe a Spinoza no sólo en el linaje de la expresión, sino además en la tradición de la univocidad: la univocidad es, a sus ojos, la clave angular de todo el espinosismo (DELEUZE, 1968, p. 88). La inmanencia, en el sentido propiamente espinosista, constituye una nueva figura de la univocidad; más aún, la inmanencia expresiva sólo es alcanzada a condición de ser unívoca (DELEUZE, 1968, pp. 58, 150, 162). Cabe subrayar que esta lectura univocizante de Spinoza es una operación propiamente deleuziana, pues el autor de la Ética no apela en ningún momento a la univocatio escotista.

Según Deleuze, la univocidad se halla en Spinoza en tres sentidos: como univocidad de los atributos, de la causa y, según el texto, de la idea (DELEUZE, 1968, p. 309) o de la modalidad (DELEUZE, 1983, p. 74). En primer lugar, la univocidad de los atributos consiste en que, bajo la misma forma, los atributos constituyen la esencia de Dios como Natura Naturante y contienen la esencia de los

R. Inter. Interdisc. INTERthesis, Florianópolis, v.11, n.2, p.15-36, Jul-Dez. 2014
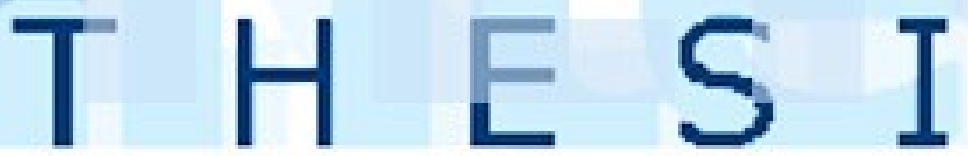
modos como Natura Naturada. En razón de ello, no hay nada común entre la esencia de Dios y la esencia de los modos, pero hay formas idénticas a Dios y a los modos: "La inmanencia significa primero la univocidad de los atributos: los mismos atributos se dicen de la substancia que ellos componen y de los modos que contienen" (DELEUZE, 1983, p. 74).

En segundo lugar, la univocidad de la causa significa que Dios es causa de todas las cosas en el mismo sentido en que es causa de sí. En otras palabras, la causa eficiente se dice en el mismo sentido en que se dice la causa de sí: "es en la inmanencia que la univocidad encontrará su fórmula propiamente espinosista: Dios se supone causa de todas las cosas en el mismo sentido (eo sensu) en que se supone causa de sí" (DELEUZE, 1968, p. 58). Deleuze le concede gran importancia al hecho de que Spinoza haya establecido como punto de partida de la Ética la definición de la causa de sí (SPINOZA, 1980, p. 29), pues

\begin{abstract}
tradicionalmente, la noción de causa de sí es empleada con muchas precauciones, por analogía con la causalidad eficiente (causa de un efecto distinto), por tanto en un sentido derivado: causa de sí significaría "como por una causa". Spinoza invierte [renverse] esta tradición y hace de la causa de sí el arquetipo de toda causalidad, su sentido originario y exhaustivo (DELEUZE, 1983, p. 77).
\end{abstract}

Desde la perspectiva de nuestro autor, la clave de la renovación espinosista de la univocidad reside en la idea de causa inmanente, en tanto ella libera a la univocidad de la neutralidad e indiferencia a las que la sometía la doctrina de la creación divina. Según Spinoza (1980, p. 47), "Dios es causa inmanente, pero no transitiva, de todas las cosas" (Deus est omnium rerum causa immanens, non vero transiens) pues es causa de todas las cosas que hay en él y no hay nada fuera.

Por último, la univocidad de la modalidad significa que lo necesario califica tanto el orden de la Naturaleza naturada como la organización de la Naturaleza naturante (DELEUZE, 1968, p. 120). Todo es necesario, ya sea por su esencia o por su causa: la Necesidad es la única afección del Ser, su única modalidad.

A juicio de Deleuze, Spinoza representa un progreso en la tradición de la univocidad pues Duns Escoto era "teólogo", lo cual significa que su preocupación por evitar el panteísmo lo llevó a concebir el ser unívoco como un concepto neutro, indiferente a lo finito y a lo infinito, a lo singular y a lo universal. En Spinoza, por el contrario, el Ser unívoco tiene un concepto determinado como "lo que se dice en un

R. Inter. Interdisc. INTERthesis, Florianópolis, v.11, n.2, p.15-36, Jul-Dez. 2014
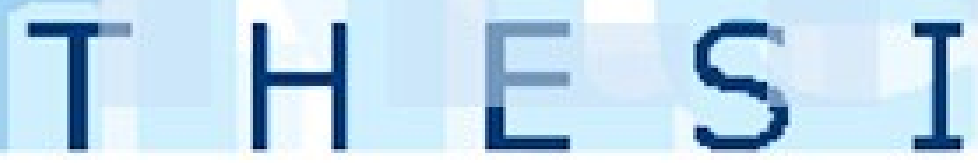
solo y mismo sentido de la substancia que es en sí y de los modos que son en otra cosa"; en otros términos, "hay una univocidad del Ser (atributos), aunque lo que es (aquello de lo cual se dice el ser) no sea lo mismo (substancia o modos)" (DELEUZE, 1983, p. 119).

Cabe destacar que, en Différence et répétition, Deleuze elabora una novedosa genealogía filosófica de la univocidad que comporta tres autores: Duns Escoto, Spinoza y Nietzsche. La particularidad de esta visión reside, por un lado, en hacer "univocistas" a Spinoza y a Nietzsche y, por otro lado, en construir una historia de rasgos teleológicos -pues la univocidad fue depurándose de una etapa a la siguiente. Deleuze señala que la historia de la filosofía presenta tres momentos en la elaboración de la univocidad del ser, el primero de los cuales corresponde a Duns Escoto. Nuestro autor manifiesta su admiración por el Opus oxoniense, al que califica como "el libro más grande de ontología pura", donde el ser es pensado como unívoco y neutro, esto es, indiferente a lo infinito y a lo finito, a lo singular y a lo universal, a lo creado y a lo increado. El "mérito" de Escoto reside en haber logrado discernir el ser más acá del entrecruzamiento entre lo universal y lo particular. No obstante, señala nuestro autor, con el objetivo de evitar el panteísmo, Escoto neutralizó el ser en un concepto abstracto: si el ser común no fuese neutro sino determinado, habría comunidad ontológica entre Dios y las criaturas. Es por ello que Deleuze afirma que Escoto se limitó a pensar el ser unívoco, ofreciendo una solución "abstracta” (cfr. DELEUZE, 2008, p. 57). En efecto, la univocidad en el Doctor Sutil no conduce al panteísmo -es decir, a la negación de la distancia entre Dios y las criaturas-, sino que convive con la analogía para salvar la teología.

El segundo momento de esta historia de la univocidad remite a Spinoza, quien operó "un progreso considerable" dado que no sólo pensó el ser unívoco sino que además lo afirmó en tanto substancia única, universal e infinita (Deus sive Natura). Según Deleuze, así como la univocidad en Duns Escoto se dirigía contra la analogía tomista, Spinoza rechazó la doctrina cartesiana de las substancias por considerarla impregnada de analogía. El "progreso" llevado adelante por Spinoza reside en negar cualquier jerarquía y eminencia, pues la substancia es igualmente designada por todos los atributos e igualmente expresada por todos los modos de acuerdo con su grado de potencia: "el ser se dice en único y mismo sentido de la substancia y de los modos, aun si los modos y la substancia no tienen el mismo sentido o no tienen este

R. Inter. Interdisc. INTERthesis, Florianópolis, v.11, n.2, p.15-36, Jul-Dez. 2014
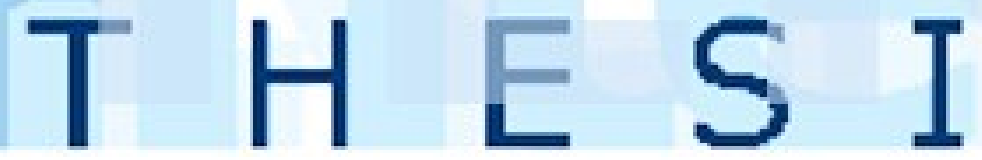
ser de la misma forma (in se e in alio)" (Deleuze, 2008, p. 59). Es por ello que el ser unívoco deja de ser neutro para devenir expresivo, "una verdadera proposición expresiva afirmativa".

\subsection{EL PROBLEMA DE LOS ATRIBUTOS: LÓGICA INMANENTE Y DISTINCIÓN FORMAL}

Venimos de ver de qué manera Deleuze inserta a Spinoza en la tradición de la univocatio con relación a los atributos, la causa y la modalidad. Nos detendremos a continuación en el primero de los sentidos, que resulta particularmente relevante en nuestro trabajo por tres razones entrelazadas: pone de relieve la oposición entre la perspectiva unívoca y la analógica; apela a la "lógica de la expresión inmanente" a fin de resolver el problema de la unidad y la multiplicidad; pone en juego el modo en que Deleuze encuentra la distinción formal escotista en Spinoza.

Nuestro autor explica que el método espinosista no procede ni por abstracción ni por analogía: los atributos no resultan de un proceso de abstracción de las cosas particulares ni son transferidos a Dios de manera analógica. Por el contrario, ellos "son directamente alcanzados como formas de ser comunes a las criaturas y a Dios, comunes a los modos y a la substancia" (DELEUZE, 1983, p. 37). Desde la perspectiva espinosista, hay a la vez "comunidad de formas" y "distinción de esencias": "la inmanencia al estado puro exige un Ser unívoco que forma una Naturaleza, y que consiste en formas positivas, comunes al productor y al producto, a la causa y al efecto" (DELEUZE, 1983, p. 157). Los atributos son formas comunes a Dios, cuya esencia constituyen, y a los modos que los implican. En otras palabras, es de la misma forma o en el mismo sentido que los cuerpos implican la extensión y que la extensión es un atributo de la substancia divina, dado que Dios no posee las perfecciones implicadas por los modos de manera diferente, eminente o superior (cf. DELEUZE, 1983, p. 39 y ss., 74).

Esta tesis es importante porque permite marcar la distancia que separa a la concepción espinoziana de la analógica: en el caso de Tomás de Aquino, las cualidades que atribuimos a Dios no implican una comunidad de forma entre Dios y las criaturas. La analogía toma ciertos caracteres de las criaturas y los atribuye a Dios de manera eminente, negando formas comunes a ambos. Deleuze señala que

R. Inter. Interdisc. INTERthesis, Florianópolis, v.11, n.2, p.15-36, Jul-Dez. 2014
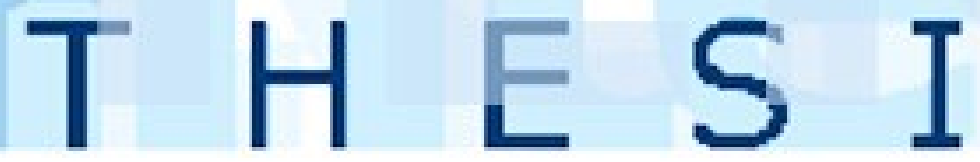
la analogía no puede sino estar acompañada de la equivocidad y la eminencia (cf. DELEUZE, 1983, p. 87; DELEUZE, 1968, p. 38).

Por otra parte, el problema de los atributos en tanto expresiones de Dios involucra la cuestión de la unidad y la multiplicidad, pues se vuelve necesario determinar cómo pueden expresiones diferentes designar una sola y misma cosa, cómo es posible que diferentes nombres designen un mismo ser. La respuesta reside en la lógica de la expresión inmanente, que Deleuze caracteriza a partir de la distinción entre aquello que una expresión -por ejemplo, una proposición- "expresa" y aquello que "designa". "Lo expresado" (l'exprimé) es el sentido de la proposición, que no existe fuera de ella, y que remite a un entendimiento que lo capta objetivamente. Lo expresado o el sentido se dice de la cosa, no de la expresión misma, pues el entendimiento lo refiere al objeto "designado". Así, los nombres de Dios se distinguen por sus sentidos, a la vez que son atribuidos al mismo objeto designado. Deleuze sostiene que Spinoza aplica este esquema a la relación entre la substancia y los atributos: cada atributo es un nombre o una expresión distinta; lo que expresa es su sentido, vinculado con la substancia en tanto objeto designado por todos los atributos; todos los sentidos expresados forman lo "expresable" o la esencia de la substancia (cf. DELEUZE, 1968, p. 53).

No obstante, nuestro autor advierte que la asimilación de la substancia al objeto designado por distintos nombres no resuelve el problema de la diferencia entre esos nombres, dado que los nombres de Dios introducen en la unidad de lo designado una multiplicidad actual. El nudo del problema reside en determinar si los atributos o nombres divinos introducen una pluralidad de quidditas en Dios. ${ }^{4}$ Con el fin de resolver la dificultad, Deleuze emplea la noción de "distinción formal" de Duns Escoto y se la atribuye a Spinoza. Reparemos en este concepto que, si bien se denominó sólo "formal" o "escotista", fue propuesto por Pedro Tomás en el siglo XIV (MAGNAVACCA, 2005, pp. 231-234).

La "distinción actual formal por la naturaleza de la cosa" (distinctio actualis formalis ex natura rei) mienta un tipo de distinción que no se halla en la cosa, como la distinción real (d. realis), ni tampoco es resultado de una operación mental, como

\footnotetext{
${ }^{4}$ La quididad es un sinónimo de la esencia, lo que una cosa es, quid est. A diferencia de otros equivalentes de la voz "essentia", quidditas alude a la esencia en cuanto es expresable en la definición de la cosa, pues esta definición nos da el "quid est" de algo. Por ejemplo, la quidditas del hombre es su humanitas, es decir su condición de animal racional. Cf. Magnavacca (2005, p. 583).
}

R. Inter. Interdisc. INTERthesis, Florianópolis, v.11, n.2, p.15-36, Jul-Dez. 2014 
la de razón (d. rationis). Antes bien, ella es "formal" porque distingue en una determinada realidad elementos que para la $d$. rationis no son distintos, y es "actual", en el sentido de real, porque se encuentra en la realidad independientemente de las operaciones lógicas; es asimismo "según la naturaleza de la cosa" porque una formalidad no incluye la otra. La distinción formal señala diferencias genuinas que existen en el mundo independientemente de la actividad del intelecto, esto es, que estarían presentes aun si no hubiera mentes que las captasen, pues la cosa misma está estructurada según la distinción. Por ejemplo, en el hombre, entre "animal" y "racional" hay una distinción formal -a diferencia del par "homo-humanitas", que es sólo una distinción de razón-: la cosa misma ya está estructurada según la diversidad del género y de la especie. En este sentido, es una distinción "real", aunque mínima, porque expresa capas de realidades que forman un ser, y al mismo tiempo no es una distinción numérica, dado que no introduce ninguna división numérica o material en el ser -v.gr., "animal" y "racional" no hacen del hombre dos seres diferentes en número (cf. DELEUZE, 1968, p. 54). De igual modo, dos atributos de Dios como la Justicia y la Bondad son nombres divinos que designan a un Dios absolutamente uno, significando al mismo tiempo quidditas diferentes.

La distinción escotista permite incluir en la definición de un ente unitario notas diversas: los ítems que son formalmente distintos tienen definiciones o rationes no idénticas. Por ejemplo, hay una distinción formal, al interior de una cosa individual, entre el género y la diferencia específica; la esencia y sus atributos propios; las facultades del alma y el alma misma; las personas de la trinidad y la esencia divina (cf. KING, 2003, pp. 9-12). En suma, la distinción formal permite compatibilizar la unidad de la sustancia y la multiplicidad de nombres mediante la aprehensión de quidditas diferentes que pertenecen a un mismo sujeto.

Ahora bien: Deleuze sostiene que la distinción real no numérica de Spinoza reproduce la distinción formal escotista. Nuestro autor explica la resolución del problema en tres pasos. Primero, los atributos en Spinoza son realmente distintos, tienen razones formales irreductibles; cada atributo expresa una esencia infinita y se distinguen formalmente. Segundo, cada atributo atribuye su esencia a la substancia "como a otra cosa". La distinción formal entre atributos no comporta división alguna en el ser. Tercero, esa otra cosa es "la misma" para los atributos: "todos los atributos formalmente distintos son relacionados por el entendimiento a una substancia 
ontológicamente una" (DELEUZE, 1968, p. 56). No se trata de un acto arbitrario, puesto que el entendimiento reproduce objetivamente la naturaleza de las formas que aprehende. Los atributos tienen identidad en el ser, distinción en la formalidad: "ontológicamente uno, formalmente diverso", tal es su estatuto.

Es conveniente reparar en una dificultad de orden exegético, similar a la ya señalada con relación a la idea de "inmanencia" -cf. supra § II. 1.-: Spinoza no utiliza el término "distinción formal". ${ }^{5}$ La tesis deleuziana reside precisamente en que el término "distinción real" empleado por Spinoza prolonga y renueva el concepto escotista. ${ }^{6}$ No obstante, para Escoto la distinción formal se ejercía sólo sobre entes de razón (géneros y especies, facultades del alma) o bien sobre los atributos divinos que, desde una perspectiva espinosista, son propiedades (justicia, sabiduría, bondad). Deleuze admite que "al proponer la imagen de un Spinoza escotista y no cartesiano, arriesgamos caer en ciertas exageraciones" (DELEUZE, 1968, p. 57). Es posible observar que la caracterización escotista de Spinoza lo posiciona claramente en el linaje contrario a la analogía, encarnado no sólo en Tomás sino también en Descartes (considerado un "heredero de Tomás"; cf. DELEUZE, 1968, p. 147):

"contra Descartes, Spinoza plantea la igualdad de todas las formas del ser, y la univocidad de lo real que se desprende de esta igualdad. De todos los puntos de vista, la filosofía de la inmanencia aparece como la teoría del Seruno, del Ser-igual, del Ser-unívoco y común" (DELEUZE, 1968, p. 152).

\subsection{EMANACIÓN VERSUS "INMANACIÓN"}

Hemos señalado que Deleuze ubica a Spinoza en el cruce de las tradiciones filosóficas de la univocidad y de la expresión, como el operador de su encuentro. Ya hemos presentado los elementos fundamentales de la primera: triple univocidad de los atributos, la causa y la modalidad; ser determinado como unívoco en cuanto se dice en un solo y mismo sentido de la substancia que es en sí y de los modos que

\footnotetext{
${ }^{5}$ Como señala Jacquet (2010), cuando Deleuze se toma ciertas "libertades" con el texto de Spinoza, empleando términos tomados de Duns Escoto que no pertenecen en rigor a Spinoza, se ocupa de aclarar que dichos conceptos no figuran literalmente. En este sentido, es posible reconocer en Deleuze a un "tesista riguroso y prudente" que no se opone a los cánones académicos que rigen la elaboración de una tesis. Al respecto, es pertinente la apreciación de Montebello (2008, p. 71), quien observa que "no se trata de imponer un sentido escotista a Spinoza sino, como en todas las lecturas cruzadas de Deleuze, de extraer un sentido por co-funcionamiento de conceptos".

6 Deleuze (1968, p. 57) argumenta que Spinoza encontró conveniente hacer uso del término "distinción real" pues ya había sido empleado por Descartes y "permitía las mayores audacias sin resucitar antiguas polémicas que Spinoza juzgaba inútiles y perjudiciales".
}

R. Inter. Interdisc. INTERthesis, Florianópolis, v.11, n.2, p.15-36, Jul-Dez. 2014
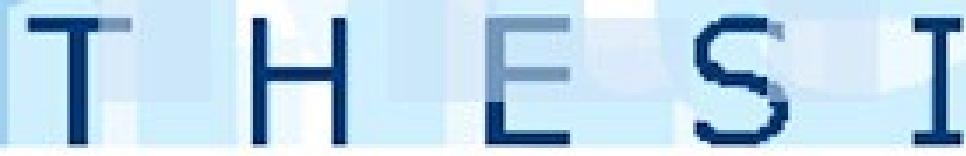
son en otra cosa; distinción formal entre la pluralidad de atributos y la unidad ontológica de la sustancia. A continuación retomaremos la comprensión deleuziana de la tradición filosófica de la expresión -cf. supra $\S$ II. 1.-, con vistas a lo cual abordaremos los lazos lógicos entre la inmanencia y la expresión, así como la formación histórica de la idea de una inmanencia expresiva.

Con respecto a la primera cuestión, Deleuze ubica su origen en el problema platónico de la participación. Entendiendo por "participar" el hecho de "tomar parte", el principio de participación es buscado del lado del participante, de modo tal que la participación aparece como una "aventura" que sobreviene del exterior al partícipe. Este esquema, explica nuestro autor, dio lugar a numerosas dificultades que los post-platónicos se propusieron resolver mediante la búsqueda de un principio que torne posible la participación desde la perspectiva del partícipe mismo. Así, en lugar de comenzar con los caracteres del participante (múltiple, sensible) para luego interrogarse cómo es posible la participación, ellos buscaron el movimiento interno que funda la participación del lado del partícipe. En el caso de Plotino, la noción de "causa emanativa" quiere resolver estas dificultades: la participación se da por emanación y consiste en una "donación productiva". La verdadera actividad está del lado del partícipe, de quien todo emana: "la causa emanativa es la causa que da, el Bien que da, la Virtud que da" (DELEUZE, 1968, p. 154).

En este marco, Deleuze compara la causa emanativa y la causa inmanente. El rasgo común reside en que ninguna sale de sí para producir, ambas permanecen en ellas mismas. Sin embargo, lo esencial es que se distinguen en la manera de producir, porque si bien la causa emanativa permanece en sí, el efecto producido no está ni permanece en ella. Por el contrario, una causa es inmanente "cuando el efecto mismo es "inmanado" (immané) en la causa en lugar de emanar de ella. Lo que define a la causa inmanente es que el efecto está en ella, sin duda como en otra cosa, pero permanece en ella" (DELEUZE, 1968, p. 156).

Este pasaje es fundamental en nuestra investigación porque muestra, mediante un deslizamiento etimológico que analizaremos a continuación, el núcleo de la renovación deleuziana de la noción espinosista de inmanencia. En efecto, venimos de ver que Deleuze distingue la causa emanativa de la causa inmanente en la medida en que, en la primera, el efecto sale de la causa, mientras que, en la segunda, el efecto permanece en ella. Si en un caso decimos que el efecto "emana"

R. Inter. Interdisc. INTERthesis, Florianópolis, v.11, n.2, p.15-36, Jul-Dez. 2014
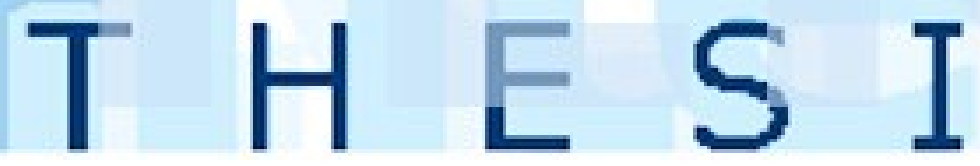
de la causa, Deleuze afirma que en el otro el efecto inmana -más precisamente, "es inmanado" (est immané)- de ella.

Ahora bien, sostener que un efecto es "inmanado" constituye una operación ilegítima desde el punto de vista etimológico pero, a la vez, una innovación conceptual. En efecto, el término inmanencia remite al verbo immanere (del griego emménõ), que es un vocablo raro del latín tardío que significa "permanecer en" (en francés: rester, résider dans). "Immanere" está compuesto por la conjunción de "in" y "manere", verbo del que toma la significación de "quedarse en el mismo lugar", "permanecer fijado" (tal como lo indican en francés demeurer, rester; en inglés, stay; en alemán, bleiben) (ERNOUT y MEILLET, 1979, pp. 383-384; FORCELLINI, 1940, pp. 170, 721; LEWIS y SHORT, 1951, p. 891; SOUTER, 1964, p. 185).

Sin embargo, Deleuze hace como si, en lugar de ser la reunión de "in" y "manere", el concepto de "inmanar" se derivase de "in" y "manare", que es un verbo con otras significaciones. En efecto, manare significa "fluir", "pasar", "derivar de"; está asociado a las operaciones de los líquidos (correr, gotear, chorrear). El verbo "emanar" deriva de manare, pero no así "inmanar", que es una invención suya. ${ }^{7}$ Cabe preguntarse, entonces, por qué acuña este vocablo. Creemos que la respuesta reside en las características de "manare" que no tiene "manere": el primero comporta una dimensión de movimiento que contrasta con la determinación del segundo a permanecer en un mismo lugar. Así, Deleuze renueva la comprensión de la causa inmanente espinoziana y propone un nuevo concepto de inmanencia definido como "inmanar", que resulta de la introducción del movimiento en el ser unívoco; en palabras de Agamben (1998, p. 172), "el ser se libera del riesgo de inercia y de inmovilidad que la absolutización de la univocidad dejaba pesar sobre él".

La segunda diferencia planteada por Deleuze entre la emanación y la "inmanación" concierne la heterogeneidad entre las filosofías del Uno y las ontologías puras. Las primeras postulan la instancia del Uno como superior al Ser, mientras que las segundas no reconocen nada por sobre el Ser (DELEUZE, 2003, pp. 244-246). Las filosofías del Uno, dominantes en la tradición platónica y neoplatónica, implican una jerarquía de los existentes, conducen a un "universo en

\footnotetext{
${ }^{7}$ El verbo "inmanar" (immaner) no existe en francés, como tampoco en español. Tanto el diccionario Petit Robert como el CNRTL [Centre National de Ressources Textuelles et Lexicales; URL=http://www.cnrlt.fr] sólo admiten los sustantivos "immanence" e "immanentisme" y el adjetivo "immanent(e)".
}

R. Inter. Interdisc. INTERthesis, Florianópolis, v.11, n.2, p.15-36, Jul-Dez. 2014 
escalera" que se sostiene a partir del Uno como principio trascendente y que procede por una serie de emanaciones y de conversiones jerárquicas. A este mundo pertenece una concepción equívoca o analógica del Ser. Nuestro autor explica que la emanación está al servicio de un universo jerarquizado: cada término es la imagen del término superior que lo precede y se define por el grado de alejamiento que lo separa de la causa primera o del primer principio.

Por el contrario, en las ontologías puras no hay un Uno superior al ser, de lo cual se sigue un "mundo de la inmanencia", anti-jerárquico, "anárquico" en la medida que todos los seres valen. No se trata, aclara Deleuze, de una fórmula nihilista del tipo "todo vale", sino de la afirmación, desde la perspectiva del ser, de que tanto la piedra como el insensato, tanto el animal como el hombre razonable, valen en tanto se dice que "son" en un mismo y único sentido -i.e. de manera unívoca. El universo entendido como "anarquía coronada" implica que todos los seres son igualmente ser, en la medida en que cada uno efectúa su propia potencia (puissance) en una vecindad inmediata con la causa primera (DELEUZE, 1981). A diferencia de la causa emanativa, que es superior al efecto, la causa inmanente plantea una igualdad del Ser, que aparece presente en todos los seres. Al contrario de lo que ocurría en el caso del universo jerarquizado, los seres no se definen por ocupar un lugar más o menos alejado del Uno, sino que cada uno depende directamente de Dios y participa en la igualdad del ser: "La inmanencia se opone a toda eminencia de la causa, a toda teología negativa, a todo método de analogía, a toda concepción jerárquica del mundo. Todo es afirmación en la inmanencia" (DELEUZE, 1968, p. 157).

Deleuze explica, a partir de los estudios de Gandillac, que en el neoplatonismo una causa de tipo inmanente se unió a la causa emanativa, lo cual dio origen a las nociones de complicare-explicare, de gran relevancia en la Edad Media y el Renacimiento. En dicho esquema, las cosas permanecen en Dios en tanto lo explican o implican, a la vez que Dios permanece en sí para complicar las cosas. Nuestro autor precisa que:

la inmanencia se define por el conjunto de la complicación y de la explicación, de la inherencia y de la implicación. Las cosas permanecen inherentes a Dios que las complica, como Dios permanece implicado por las cosas que lo explican [...] la inmanencia se revela expresiva, la expresión inmanente, en un sistema de relaciones lógicas en el que ambas nociones son correlativas" (DELEUZE, 1968, p. 159, s.n.)

R. Inter. Interdisc. INTERthesis, Florianópolis, v.11, n.2, p.15-36, Jul-Dez. 2014
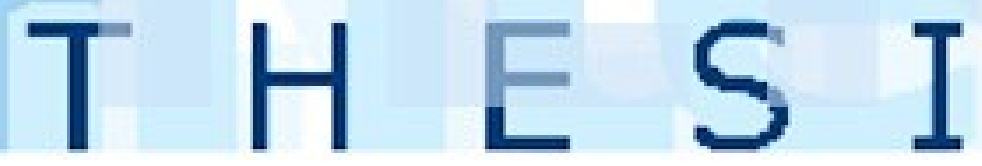
Deleuze considera, retomando los análisis de Koyré, que la especificidad de la filosofía del Renacimiento reside en la categoría de expresión (cf. DELEUZE, 1968, pp. 12, 161). La tesis de nuestro autor, insistimos, es que la tendencia expresionista en la filosofía occidental no fue realizada por completo hasta Spinoza; su larga historia filosófica es "un poco oculta, un poco maldita" a causa de su orientación panteísta (DELEUZE, 1968, p. 229). Si bien admite que la inmanencia expresiva aparecía tanto en la temática de la emanación como en la creación, en ambas permanecía subordinada a las demandas de la trascendencia:

los temas de la creación o de la emanación no pueden prescindir de un mínimo de trascendencia, que le impide al "expresionismo" ir hasta el fondo de la inmanencia que implica. La inmanencia es precisamente el vértigo filosófico, inseparable del concepto de expresión (doble inmanencia de la expresión en lo que se expresa, y de lo expresado en la expresión) (DELEUZE, 1968, pp. 163-164, las cursivas son nuestras)

Deleuze sostiene que, si bien el cristianismo favorece la tendencia expresionista a causa de su teoría del Verbo y por hacer del primer principio un ser, la exigencia de mantener la trascendencia del ser divino lo llevó a rechazarla; de allí la constante "acusación de inmanencia y de panteísmo" con que se amenazaba a los filósofos. La causa inmanente estaba presente antes de Spinoza, pero nunca lo hacía "hasta el límite" porque implicaba el "peligro", la herejía filosófica de negar la diferencia entre la causa y el efecto -la acusación de inmanentista reside precisamente en confundir a Dios y a las criaturas. Era imperioso, desde la perspectiva de la expresión, "liberar" a la causalidad inmanente de su subordinación a otras causalidades, lo cual es consumado, según Deleuze, al conjugarse con la tradición de la univocidad iniciada por Duns Escoto: "la inmanencia expresiva no puede bastarse a sí misma mientras no se acompañe de una plena concepción de la univocidad, de una plena afirmación del Ser unívoco" (cf. DELEUZE, 1968, pp. 161162). De donde se sigue la importancia de Spinoza:

"el significado del espinosismo nos parece el siguiente: afirmar la inmanencia como principio; despejar la expresión de toda subordinación al respecto de una causa emanativa o ejemplar. La expresión misma deja de emanar, como de reunir. Ahora bien, tal resultado no puede ser obtenido sino en una perspectiva de univocidad" (DELEUZE, 1968, p. 164).

R. Inter. Interdisc. INTERthesis, Florianópolis, v.11, n.2, p.15-36, Jul-Dez. 2014
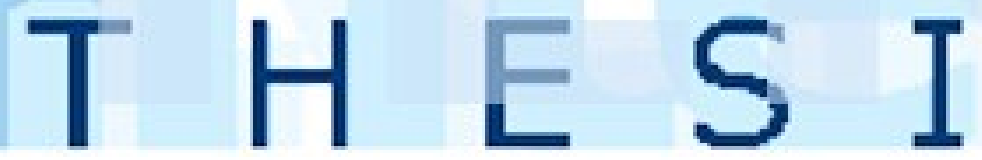


\section{CONCLUSIÓN}

En este artículo hemos desarrollado el origen de la idea de inmanencia en Deleuze. Esta noción aparece por primera vez en Spinoza et le problème de l'expression de dos maneras entrelazadas. Por un lado, la inmanencia caracteriza el conjunto de la filosofía expresionista de Spinoza, si bien hemos observado que el autor de la Ética no emplea el término como tal, en su forma sustantivada, sino sólo como adjetivo que califica un tipo de causalidad. Por otro lado, nuestro autor acuña el giro "ser inmanado" al momento de describir la especificidad de la causa inmanente con relación a la causa emanativa.

Hemos desarrollado el marco más general, que remite al tratamiento deleuziano de la tradición expresionista en filosofía. La idea de expresión tiene por consecuencia el panteísmo, entendido como la inmanencia de la expresión en lo que se expresa y de lo expresado en la expresión. No obstante, la expresión ha oscilado en la historia de la filosofía entre concepciones ligadas a la creación y a la emanación que, por contener un elemento de trascendencia, impedían la inmanencia radical. La tesis de Deleuze es que la inmanencia expresiva fue alcanzada cuando la vertiente expresionista se conjugó con la univocidad. De allí que argumente que encuentra en Spinoza conceptos de Duns Escoto.

Hemos visto que el primer elemento escotista puesto en juego concierne a la univocidad del ser. Deleuze sostiene que el ser es unívoco porque se dice en el mismo sentido de la sustancia que es en sí y de los modos que son en otra cosa, de la Naturaleza como Natura Naturante y como Natura Naturada. Nuestro autor halla una triple univocidad en el espinosismo: los atributos, la causa y la modalidad. El primer rasgo se dirige contra Descartes, para quien Dios es causa de sí pero en un sentido eminente, analógico, diferente a como es causa de sí, mientras que Spinoza la convirtió en el arquetipo de la causa, su sentido originario.

Hemos señalado que la posición cartesiana es deudora de la tomista, y la univocidad de los atributos se opone precisamente a Tomás de Aquino. Para éste, el conocimiento por analogía parte del dato sensible y luego le atribuye las características de las criaturas a Dios en grado eminente. Spinoza, por el contrario, considera que los atributos son formas comunes a Dios y a las criaturas. La lógica de la inmanencia espinosista hace de los atributos la expresión unívoca de Dios y de

R. Inter. Interdisc. INTERthesis, Florianópolis, v.11, n.2, p.15-36, Jul-Dez. 2014
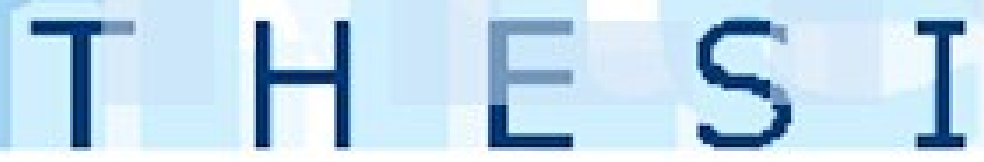
las criaturas. La multiplicidad de atributos y nombres divinos es compatibilizada con la unidad ontológica mediante el recurso a la noción escotista de distinción formal, que permite aprehender quidditas diferentes en un mismo sujeto. Si bien ella no es empleada por Spinoza, Deleuze argumenta que la distinción real no numérica espinosista corresponde al concepto de Escoto.

Hemos puntualizado que la primera aparición de la idea de inmanencia en la obra de nuestro autor requiere dos precisiones de índole filológica. Por un lado, Deleuze argumenta que la filosofía espinosista es una filosofía de la "inmanencia" cuando, en rigor, Spinoza no emplea jamás el sustantivo en cuestión, sino que hace uso del adjetivo para calificar la causalidad "inmanente". Aun si es legítimo afirmar que la idea está presente en el pensamiento espinosista, el término en cuanto tal pertenece a la lectura deleuziana.

Por otro lado, Deleuze reelabora el concepto de "inmanencia" al sostener que lo propio de la causa inmanente es que el efecto resulta "inmanado" en la causa, en lugar de ser "emanado". Como hemos visto, se trata de un deslizamiento etimológico que va del vocablo latín manere, que es el auténtico origen del término, a manare, que es el verbo del cual deriva "emanar" y que connota movimiento. El objetivo de esta estrategia filosófica es doble: por un lado, busca acentuar la proximidad y a la vez la distancia entre la causa emanativa y la causa inmanente, como si "emanar" e "inmanar" fuesen actos comparables -cuando, en verdad, el segundo es una invención conceptual de nuestro autor-; por otro lado, tiende a dotar de movimiento a la causa inmanente, a imprimirle una connotación ligada a lo que fluye.

Hemos observado también las implicancias de los tipos de causalidad. La causa emanativa da lugar a filosofías del Uno organizadas en torno a un principio trascendente, superior al ser, con relación al cual se establece una jerarquía de seres repartidos según su distancia con el arkhé. La causa inmanente está a la base de ontologías puras, donde el Uno no trasciende el ser y todos los seres valen por igual en cuanto efectúan su potencia. Hemos marcado también que la causa inmanente se distingue de la causa analógica de Tomás de Aquino, quien concibe a Dios en términos de causae agentis analogice: no es una causa unívoca puesto que la semejanza entre la causa -el creador- y sus efectos -las criaturas- no es perfecta; la perfección es comunicada sólo de manera parcial y disminuida. El Aquinate argumenta que Dios es un agens analogicum: Dios como causa habita lo más íntimo

R. Inter. Interdisc. INTERthesis, Florianópolis, v.11, n.2, p.15-36, Jul-Dez. 2014
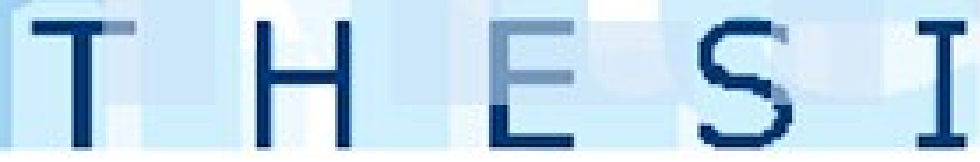
de todas sus criaturas, sin dejar por ello de trascenderlas. A la luz de lo expuesto, se hace patente la contraposición entre la desigualdad esencial entre la causa y el efecto, en el caso de la causa analógica, y la igualdad, enfatizada por Deleuze, entre la causa inmanente y su efecto. Este rasgo de igualdad del mundo de la inmanencia anula cualquier trascendencia.

R. Inter. Interdisc. INTERthesis, Florianópolis, v.11, n.2, p.15-36, Jul-Dez. 2014
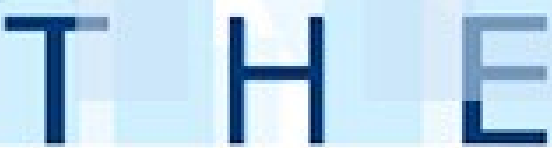


\title{
SOBRE A ORIGEM DO CONCEITO DE IMANÊNCIA EM GILLES DELEUZE
}

\section{Resumo:}

O conceito de imanência tem uma importância fundamental na filosofia de Gilles Deleuze. Ainda quando a sua noção de "plano de imanência" e realçada a miúdo, o pensador francês desenvolveu uma aproximação integral do conceito, fazendo uma conexão com os problemas políticos (o capitalismo e descrito como um sistema imanente) e os éticos (a moralidade universal e substituída por uma ética de valores imanentes). No entanto, a primeira apresentação do conceito de imanência em seu trabalho não a sido ainda estudado em profundidade. Portanto, a nossa proposta e analisar os aspetos filosóficos e filológicos do contexto da origem da ideia de imanência em Deleuze. Baseado na nossa hipóteses, a primeira apresentação do conceito e achado na leitura de Deleuze de Spinoza em particular no seu livro Spinoza et le problème de l'expression (1968), onde nosso autor apresenta a Spinoza na tradição do univocatio entis começado por Duns Scotus. Nós mantemos que a imanência deleuziana deriva de duas perspectivas: a tesis scotista univocatio em contraste com a analogia entis da filiação tomista e a causa de Spinoza sui em contraste com a causa emanativa neoplatônica e a causa analógica tomista. A fórmula "Imanência = Univocidade", acentuada por Badiou, expressa este nó conceitual, que nos desenvolveremos. Desde o ponto de vista filológico, nos daremos prova de que Deleuze inventou o termo "ser imanado" para descrever a especificidade da causa imanente, desde uma utilização heterodoxa dos verbos em latim manare e manere.

Palavras-chave: Deleuze. Duns Scotus. Spinoza. Imanência. Univocidade.

\section{ON THE ORIGIN OF THE CONCEPT OF IMMANENCE IN GILLES DELEUZE}

\begin{abstract}
:
The concept of Immanence plays a fundamental role in the philosophy of Gilles Deleuze. While his notion of "plan of immanence" is often underlined, the French thinker developed a comprehensive approach on this concept, connecting it with political problems (capitalism is described as an immanent system) and ethical (universal morality is replaced by an ethics of immanent values). However, the first appearance of the concept of immanence in his work has not been thoroughly studied. Therefore, we propose the analysis of conceptual and philological aspects of the context of the origin of the deleuzian idea of immanence. According to our hypothesis, the first occurrence of the concept is situated in Deleuze's reading of Spinoza, particularly in Spinoza et le problème de l'expression (1968), where our author inscribed Spinoza in the tradition of the univocatio entis initiated by Duns Scotus. We hold that the Deleuzian immanence derives from two perspectives: the scotist thesis of the univocatio against the analogia entis of thomistic filiation and the Spinozian cause sui against the Neoplatonist emanative cause and the thomistic analogical cause. The formula "Immanence = univocity", emphasized by Badiou, expresses this conceptual knot that we will develop. From the philological point of view, we will show that Deleuze coined the term "to be immanated", at the time of describing the specificity of the immanent cause, from a heterodox use of the Latin verbs manare and manere.
\end{abstract}

Keywords: Deleuze. Duns Scot. Spinoza. Immanence. Univocity.

R. Inter. Interdisc. INTERthesis, Florianópolis, v.11, n.2, p.15-36, Jul-Dez. 2014
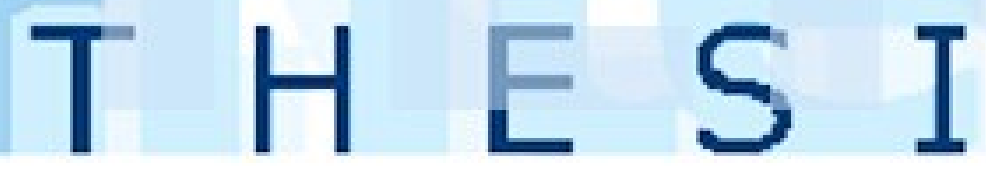


\section{REFERENCIAS}

ABBAGNANO, Nicolás. Diccionario de Filosofía. FCE: México, 1995.

AGAMBEN, Giorgio. L'immanence absolue. In: ALLIEZ, Eric (Ed.). Gilles Deleuze. Une vie philosophique. Paris: Synthélabo, 1998. P. 165-188.

ARISTÓTELES. Metafísica. Edición trilingüe (griego, latín, castellano) por Valentín García Yebra. Madrid: Gredos, 1998.

BADIOU, Alain. Deleuze, «La clameur de l'Etre». Paris: Hachette, 1997.

BADIOU, A. De la vie comme nom de l'Être. Rue Descartes : « Gilles DeleuzeImmanence et vie », Paris, $n^{\circ}$ 20, PUF, pp. 27-34, 1998.

Centre National de la Recherche Scientifique. Centre National de Ressources Textuelles et Lexicales. Disponible en : http://www.cnrtl.fr/

CICCARELLI, Roberto. Immanenza. Filosofia, diritto e politica della vita dal XIX al XX secolo. Bologna: II Mulino, 2008.

DE BEISTEGUI, M. The Vertigo of immanence: Deleuze's spinozism. Research in Phenomenology, The Netherlands, $n^{\circ}$ 35, 2005.

DELEUZE, Gilles. Différence et répétition. Paris: P.U.F., 2008.

DELEUZE, Gilles. Deux régimes de fous. Textes et entretiens 1975-1995. Edición de D. Lapoujade. Paris : Minuit, 2003.

DELEUZE, Gilles. Spinoza. Philosophie pratique. Paris : Minuit, 1983.

R. Inter. Interdisc. INTERthesis, Florianópolis, v.11, n.2, p.15-36, Jul-Dez. 2014
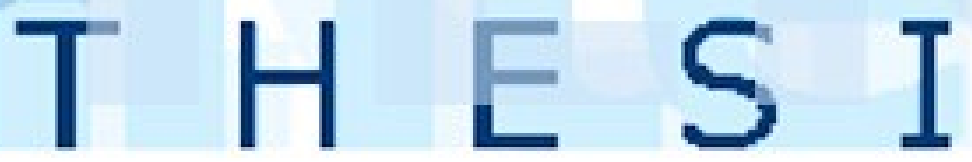
DELEUZE, Gilles. Spinoza et le problème de l'expression. Paris : Minuit, 1968.

DELEUZE, Gilles. Cours Vincennes 24/3/81. Disponible en :

http://www.webdeleuze.com/php/texte.php?cle=45\&groupe=Spinoza\&langue=1

DELEUZE, Gilles; GUATTARI, Félix. L’Anti-CEdipe. Capitalisme et Schizophrénie 1. Paris : Minuit, 1973.

DELEUZE, Gilles ; GUATTARI, Félix. Mille Plateaux. Capitalisme et Schizophrénie 2. Paris : Minuit : 2006.

ERNOUT, Alfred ; MEILLET, Alfred. Dictionnaire étymologique de la langue latine. Histoire des mots. Paris : Klincksieck, 1979.

FORCELLINI, Aegidii. Totius Latinitatis Lexicon. Leiden, 1940.

HARDT, Michael. Gilles Deleuze. An Apprenticeship in Philosophy. University of Minnesota, 1993.

JACQUET, Ch. Deleuze y su lectura conjunta de Spinoza y de Nietzsche. Instantes y azares. Escrituras nietzscheanas, Buenos Aires, Año VII, Nos 4-5, 2007.

KERSLAKE, Christian. Immanence and the vertigo of philosophy: from Kant to Deleuze. Edinburgh : Edinburgh University Press, 2009.

KING, Peter. Duns Scotus on metaphysics. In: AAVV. The Cambridge Companion to Duns Scotus. Cambridge : Cambridge University Press, 2003. P. 15-68.

LALANDE, André. Vocabulaire technique et critique de la philosophie. Paris : PUF, 1968.

LEWIS, Charlton; SHORT, Charles. A Latin dictionary. Oxford: Clarendon Press, 1951.

R. Inter. Interdisc. INTERthesis, Florianópolis, v.11, n.2, p.15-36, Jul-Dez. 2014
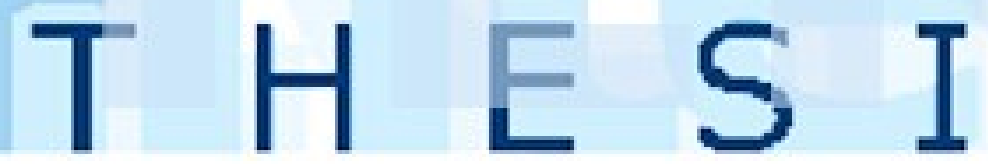
MACHEREY, P. The encounter with Spinoza. In: PATTON, Paul (Ed.). Deleuze: a critical reader. Cornwall: Blackwell, 1996. P. 139-161.

MAGNAVACCA, Silvia. Léxico técnico de filosofía medieval. Bs. As.: Miño y Dávila, 2005.

MENGUE, Philippe. Gilles Deleuze ou le système du multiple. Paris : Kimé, 1994.

MONTEBELLO, Pierre. Deleuze. La passion de la pensée. Paris : Vrin, 2008.

SHIRANI, Takashi. Deleuze et une philosophie de l'immanence. Paris: L'Harmattan, 2007.

SMITH, Daniel. Essays on Deleuze. UK: EUP, 2012.

SOUTER, Alexander (Comp.). A glossary of Later Latin to 600 AD. Oxford: Clarendon Press, 1964.

SPINOZA, Baruch. Ética demostrada según el orden geométrico. Madrid: Ediciones Orbis, 1980.

Artigo:

Recebido em Janeiro de 2014

Aceito em Junho de 2014

R. Inter. Interdisc. INTERthesis, Florianópolis, v.11, n.2, p.15-36, Jul-Dez. 2014 